\title{
Cancer stem cells and microglia in the processes of glioblastoma multiforme invasive growth
}

\author{
IGOR BRYUKHOVETSKIY ${ }^{1,2}$, IGOR MANZHULO ${ }^{1,2}$, POLINA MISCHENKO $^{1,2}$, ELENA MILKINA $^{1,2}$, \\ INESSA DYUIZEN $^{1,2}$, ANDREY BRYUKHOVETSKIY ${ }^{1,3}$ and YURI KHOTIMCHENKO ${ }^{1,2}$ \\ ${ }^{1}$ Laboratory of Molecular and Cellular Neurobiology, School of Biomedicine, Far Eastern Federal University, \\ Vladivostok 690091; ${ }^{2}$ Laboratory of Pharmacology, A.V. Zhirmunsky Institute of Marine Biology, \\ Far Eastern Branch of the Russian Academy of Sciences, Vladivostok 690059; \\ ${ }^{3}$ NeuroVita Clinic of Restorative and Interventional Neurology and Therapy, Moscow 115478, Russia
}

Received August 3, 2015; Accepted June 16, 2016

DOI: $10.3892 / \mathrm{ol} .2016 .4886$

\begin{abstract}
The development of antitumor medication based on autologous stem cells is one of the most advanced methods in glioblastoma multiforme (GBM) treatment. However, there are no objective criteria for evaluating the effectiveness of this medication on cancer stem cells (CSCs). One possible criterion could be a change in the number of microglial cells and their specific location in the tumor. The present study aimed to understand the interaction between microglial cells and CSCs in an experimental glioblastoma model. C6 glioma cells were used to create a glioblastoma model, as they have the immunophenotypic characteristics of CSCs. The glioma cells $\left(0.2 \times 10^{6}\right)$ were stereotactically implanted into the brains of 60 rats. On the 10th, 20th and 30th days after implantation, the animals were 15 of the animals were sacrificed, and the obtained materials were analyzed by morphological and immunohistochemical analysis. Implantation of glioma cells into the rat brains caused rapid development of tumors characterized by invasive growth, angiogenesis and a high rate of proliferation. The maximum concentration of microglia was observed in the tumor nodule between days 10 and 20; a high proliferation rate of cancer cells was also observed in this area. By day 30, necrosis advancement was observed and the maximum number of microglial cells was concentrated in the invasive area; the invasive area also exhibited positive staining for CSC marker antibodies. Microglial cells have a key role in the invasive growth processes of glioblastoma, as demonstrated by the location of CSCs in the areas of microglia maximum concentration. Therefore, the present study indicates that changes in microglia position and corresponding suppression
\end{abstract}

Correspondence to: Dr Igor Bryukhovetskiy, Laboratory of Molecular and Cellular Neurobiology, School of Biomedicine, Far Eastern Federal University, 8 Sukhanova Street, Vladivostok 690091, Russia

E-mail: igbryukhovetskiy@gmail.com

Key words: glioblastoma, cancer stem cells, stem cells, microglia of tumor growth may be objective criteria for evaluating the effectiveness of biomedical treatment against CSCs.

\section{Introduction}

The existing approaches for glioblastoma multiforme (GBM) treatment are not very effective. Despite the efforts of oncologists, only $10 \%$ of patients survive $>18$ month after diagnosis, and 5-year survival is almost nonexistent. The median survival time of patients with GBM is $12-15$ months (1). One of the reasons that traditional treatment procedures are ineffective is tumor invasive growth caused by the high activity rate of cancer stem cells (CSCs). This invasive process is combined with the high infiltration rate of tumor cells into brain parenchyma, meaning that radical resection of the tumor cannot be performed. This is primary reason for relapse and prolonged tumor growth (1-3). Traditional treatment methods, which include maximal surgical resection followed by a combination of radiotherapy and chemotherapy (usually temozolomide), have almost no effect on tumor cells infiltrating the brain parenchyma, and there are currently almost no medication or technologies that are able to eliminate CSCs effectively. One of the possible ways to improve the treatment of glioblastoma is to develop biomedical drugs that are able to control the proliferative and reproductive functions of CSCs. The development of such medication is more successful when using patients' own stem cells (SCs) as a basic material (4-6).

SCs have been used in the complex treatment of malignant tumors for $>50$ years. The leading clinics of Russia, Europe and the USA have perfected a method of SC extraction from the peripheral blood of patients (1). The cell concentrate is based on separated mononuclear cells with a high rate of hematopoietic SCs $\left(\mathrm{CD} 34^{+} / \mathrm{CD} 45^{+}\right)$. The sphere of their application is not limited to blood system recovery following high-dose chemotherapy; they are widely used for treating acute diseases and traumas of the nervous system, and may be of great interest in neuro-oncology $(7,8)$.

After being introduced into the vasculature, SCs are able to migrate in a certain direction until they reach the tumor nidus in the brain. The main source of chemo-attractants for SCs is damaged nervous tissue and tumor cells themselves. Previous 
studies have identified $>80$ cytokines, growth factors and other ligands controlling SC migration processes via their corresponding type of sensory receptors $(4,5,9)$. In the nidus, SCs can exhibit both an antagonistic and stimulating influence on the tumor. However, no objective criteria have been suggested that allow evaluation of the impact of cell transplantation on the neoplastic process. The most useful criterion of evaluating this medication effect on CSCs may be qualitative and quantitative changes that microglia undergo.

First, the transplantation of autologous SCs extracted from patients' peripheral blood enables the transformation of monocytes into microglia elements of the tumor that have an ambiguous role in invasive growth processes. Second, microglial cells release a large amount of $\mathrm{H}_{2} \mathrm{O}_{2}, \mathrm{NO}$ and other cytokines that damage nervous tissue (6). Microglial cell cultivation together with glioblastoma CSCs intensifies these abilities, supporting the hypothesis of a controversial association between microglia and CSCs (10). Finally, a type of microglia that counteracts invasive tumor development has been identified (11). It is obvious that histophysiological evaluation of the influence of microglial cells on glioblastoma growth will become a crucial factor in understanding the way that cell culture modification occurs and allow criteria to be proposed for defining the efficiency of biomedical cell-based treatment.

The present study aimed to understand the nature of interaction between microglial cells and CSCs in an experimental glioblastoma model in vivo.

\section{Materials and methods}

Ethical approval. The study was approved by the Ethics Committee of the School of Biomedicine, Far Eastern Federal University (Vladivostok, Russia; Minutes of Meeting No. 1 of February 2, 2015).

Equipment. Slides were analyzed using an Axio Scope A1 microscope (Carl Zeiss AG, Jena, Germany), a FluoView FV 1200MPE (Olympus Corporation, Tokyo, Japan) for deeper imaging of immunohistochemically stained biomaterials, and a Zeiss LSM 710 META confocal (laser scanning) microscope (Carl Zeiss AG) for histology.

Animals. Mature (3-4 months old) female Wistar rats $(n=60)$ weighing 200-220 g were obtained from Charles River Laboratories (Erkrath, Germany) and maintained at our facility at $24^{\circ} \mathrm{C}$ with standard atmospheric conditions, and fed with standard pellet rat feed and tap water as drinking water. On every 10th day of the experiment, 15 rats were sacrificed following C6 injection. Sacrifice was conducted as follows: Rats were anesthetized by intraperitoneal injection with a mixture of Zoletil (Virbac, Carros, France) and Rometar (Bioveta, Ivanovice na Hané, Czech Republic) at a ratio of 1:4 (10 mg/kg). Once they reached a surgical level of anesthesia, their hearts were surgically exposed. Following incision to the right atrium, the left ventricle was injected with $10 \mathrm{ml}$ cold $0.9 \% \mathrm{NaCl}$ solution followed by $20 \mathrm{ml}$ cold fixative (4\% paraformaldehyde in $0.1 \mathrm{M}$ phosphate buffer, $\mathrm{pH}$ 7.2).

C6 glioma cell line. The C6 glioma cell line (CCL-107; American Type Culture Collection, Manassas, VA, USA) was used in the present study. In comparison with other experimental glioma tumors (CNS1, RT-2, BT4C, F98, RG2, T9), C6 has the largest proportion of CSCs (12). The cells were cultivated at $37^{\circ} \mathrm{C}$ with $5 \% \mathrm{CO}_{2}$ in $25-\mathrm{cm}^{2}$ vials filled with Dulbecco's modified Eagle medium (DMEM) medium containing 10\% fetal bovine serum (FBS) and Antibiotic-Antimycotic (100X), containing 10,000 IU/ml penicillin, $10,000 \mu \mathrm{g} / \mathrm{ml}$ streptomycin and $25 \mu \mathrm{g} / \mathrm{ml}$ fungizone (Gibco; Thermo Fisher Scientific, Inc., Waltham, MA, USA). The culture medium was renewed every 3 days. After reaching 95\% confluence, the cells were treated with TrypLE Express Enzyme solution (Gibco; Thermo Fisher Scientific, Inc.; $12604-021$ ) for $7 \mathrm{~min}$ at $37^{\circ} \mathrm{C}$, placed into a $15-\mathrm{ml}$ vial and centrifuged for $3 \mathrm{~min}$ at $120 \mathrm{x}$ g. The supernatant was removed, DMEM + 10\% FBS + 100X Antibiotic-Antimiotic fresh medium was added to the pellet and the culture was transferred into a new $25-\mathrm{cm}^{2}$ culture vial.

Following suspension in fresh medium, the cells were counted using a hemocytometer and their viability was determined by staining with $0.4 \%$ trypan blue (Gibco; Thermo Fisher Scientific, Inc.; 15250-061). Prior to implantation into rat brains, C6 cells were assigned immunocytochemical characteristics, as follows.

Immunocytochemical characteristics of C6 glioma cells. Cells were fixed with $4 \%$ paraformaldehyde (Sigma-Aldrich, St. Louis, MO, USA; P6148) for $20 \mathrm{~min}$ at $4^{\circ} \mathrm{C}$ then washed 3 times for 10 min each with PBS pH 7.4 (0.1 M; cat no. 10010031; Gibco; Thermo Fisher Scientific, Inc.). Cells were then treated with 0.2\% Tween-20 (cat no. P9416; Sigma-Aldrich), permeabilized with $0.2 \%$ Triton X-100 (cat no. T8787; Sigma-Aldrich) and blocked with $0.3 \%$ bovine serum albumin (cat no. A2058; Sigma-Aldrich) (15 min incubation for each reagent; room temperature), followed by washing with PBS working solution.

Staining was performed using the following primary antibodies: p53 mouse (dilution, 1:100; cat no. AHO0152; Novex; Thermo Fisher Scientific, Inc.), nestin rabbit (dilution, 1:100; cat no. 5413; Sigma-Aldrich), glial fibrillary acidic protein mouse (dilution, 1:50; cat no. 7260; Abcam, Cambridge, UK), $\beta$ III tubulin mouse (dilution, 1:100; cat no. 7751; Abcam), S100 rabbit (cat no. 868; Abcam; dilution, 1:100) and C-X-C chemokine receptor type 4 (CXCR4) rabbit (cat no. 2074; Abcam; dilution, 1:100) antibodies. All primary antibodies were incubated for $18 \mathrm{~h}$ at $4^{\circ} \mathrm{C}$. Cells were then washed with PBS working solution 3 times for 10 min each and incubated with anti-rabbit secondary antibodies conjugated with Alexa 633 (dilution, 1:500; cat no. A21071) or Alexa 488 (Novex; cat no. A11029; Thermo Fisher Scientific, Inc.; dilution, 1:500) for $2 \mathrm{~h}$ at $37^{\circ} \mathrm{C}$. Subsequently, the cells were washed with PBS working solution 3 times for $10 \mathrm{~min}$ each. Nuclei were contrasted with DAPI (cat no. D1306; Molecular Probes; Thermo Fisher Scientific, Inc.) for $7 \mathrm{~min}$ at $22^{\circ} \mathrm{C}$, washed with PBS working solution 2 times for 10 min and placed in Mowiol 40-88 (cat no. 324590; Sigma-Aldrich). Secondary antibodies Alexa 488 and Alexa 633 were also used alone without primary antibodies as controls. Primary and secondary antibodies were used according to the manufacturer's protocol.

Glioblastoma modeling. Animals were anesthetized by injection with a 1:4 ratio mixture of Zoletil 100 and Rometar at a dose of $10 \mathrm{mg} / \mathrm{kg}$. Soft tissues of the head were dissected in 
sterile conditions, according to previously determined coordinates, and a burr hole was made with a micro drill $(1.0 \mathrm{~mm}$ diameter; Harvard Bioscience, Inc., Holliston, MA, USA).

Using stereotaxic instruments for rats (Nirishige Ltd., Tokyo, Japan) and rat brain atlas coordinates (anterior, interaural $2.4 \mathrm{~mm}$; lateral, $3.0 \mathrm{~mm}$; ventral, $4.5 \mathrm{~mm}$ ) (13), $2 \times 10^{5} \mathrm{C} 6$ glioma cells were introduced into the caudoputamen in $20 \mu \mathrm{l}$ DMEM with a Hamilton syringe (Thermo Fisher Scientific, Inc.) at a rate of $2 \mu \mathrm{l} / \mathrm{min}$. Tumor development was monitored once per week by MRI on a BioSpec scanner (Bruker, Billerica, MA, USA). On the 10th, 20th and 30th days after implantation, 15 animals were sacrificed, while the remaining animals were left to assess survivability.

Histological staining. Rats were anesthetized by intraperitoneal injection with a mixture of Zoletil 100 (Virbac) and Rometar (Bioveta) at a ratio of 1:4 (10 mg/kg) and transcardially perfused with $10 \mathrm{ml}$ cold $0.9 \% \mathrm{NaCl}$ saline followed by $20 \mathrm{ml}$ cold fixative (4\% paraformaldehyde in $0.1 \mathrm{M}$ phosphate buffer, $\mathrm{pH}$ 7.2). The brains were immediately removed and post-fixed for $12 \mathrm{~h}$ at $4^{\circ} \mathrm{C}$ in fresh buffered $4 \%$ paraformaldehyde. After rinsing, the brains were processed and embedded in paraffin, according to standard embedding techniques. Coronal cuts of the paraffin-embedded brain were made until the tumor was exposed, after which $7-\mu \mathrm{m}$ slices were obtained using a microtome. All three areas of interest (tumor, near-tumor area and symmetric area of the opposite hemisphere) were present on each slide. The near-tumor area was defined as the $200-\mu \mathrm{m}$ of tissue around the tumor edge. Paraffin-embedded brain sections $(7 \mu \mathrm{m})$ were stained with hematoxylin-eosin (Bio Optica, Milano, Italy), according to the standard procedure for morphological tissue analysis.

Immunohistochemistry. Paraffinized sections of the brain (7 $\mu \mathrm{m}$ ) were deparaffinized and incubated in $3 \%$ hydrogen peroxide to block endogenous peroxidase prior to immunohistochemical staining. After three washes in $0.1 \mathrm{M}$ phosphate buffer ( $\mathrm{pH} 7.2$ ), sections were treated for $60 \mathrm{~min}$ in a $2 \%$ bovine serum albumin solution (cat no. SC-2323; Santa Cruz Biotechnology, Inc., Dallas, TX, USA) and 0.25\% Triton X-100 (GERBU Biotechnik GmbH, Heidelberg, Germany). Brain sections were then incubated with primary antibodies on glass slides in a humidified chamber at $4^{\circ} \mathrm{C}$ for $24 \mathrm{~h}$. After three washes with $0.1 \mathrm{M}$ phosphate buffer $(\mathrm{pH} 7.2)$, the sections were incubated in a secondary antibody solution for $60 \mathrm{~min}$. Primary mouse monoclonal antibodies against proliferating cell nuclear antigen (PCNA; dilution, 1:2,000; cat no. ab29; Abcam) and transforming growth factor- $\beta 2$ (TGF- $\beta 2$; dilution, 1:100; cat no. ab36495; Abcam); and rabbit polyclonal antibodies against ionized calcium-binding adapter molecule-1 (IBA-1; dilution, 1:500; cat no. ab108539; Abcam), nestin (dilution, 1:100; cat no. N5413; Sigma-Aldrich), CXCR4 (dilution, 1:500; cat no. ab2074; Abcam) and interleukin-1 $\beta$ (IL-1 $\beta$; dilution, 1:1,000; cat no. ab9722; Abcam) were used. Appropriate anti-rabbit (cat no. PI-1000) and anti-mouse (cat no. PI-2000) secondary antibodies conjugated to horseradish peroxidase were used, according to the manufacturer's instructions (dilution, 1:100; Vector Laboratories, Inc., Burlingame, CA, USA). Negative controls were treated with PBS instead of primary antibody. After washing with $0.1 \mathrm{M}$ phosphate buffer ( $\mathrm{pH}$ 7.2), sections were treated for 5-10 min with two chromogens, DAB Plus (Thermo Fisher Scientific, Inc.) and NovaRED (Vector Laboratories, Inc.), to elicit the immunoperoxidase reaction. The sections were washed with $0.1 \mathrm{M}$ phosphate buffer (pH 7.2), dehydrated and embedded in Toluene-Free Mounting Medium (cat no. CS705; Dako, Glostrup, Denmark).

Image analysis. The number of PCNA-immunopositive cells was calculated as a proportion of the total GBM area using the ImageJ software package (version 1.41; National Institutes of Health, Bethesda, MD, USA). The Scion Image software package (version 4.0.3; Scion Corporation, Frederick, MD,USA) was used to determine the immunohistochemically stained area of microglia/macrophage elements in sections of rat brain using staining of IBA-1. Different areas of the brain preparations were captured for image analysis, with at least 80 images captured in each group of animals. Following image acquisition, all images were saved in the TIF format (1,080x800 pixels). Micrograph processing included the following steps: Converting the image to black and white, background subtraction, contrast enhancement, binarization, noise reduction and measurement. Image preprocessing included calibration (pixels translated to $\mathrm{mm}^{2}$ ) and the first three steps of micrograph processing. Per-pixel image background subtraction was used to adjust image brightness. The standard morphological filters Erode and Dilate were used to reduce background noise. Erode removes background irregularities as well as pieces of the cell soma and processes that extend beyond the slice thickness. Dilate returns the cell area to a value comparable to the area before applying the Erode filter. Image binarization was necessary to correct filter operations and the area of objects (positive staining) present in the area of interest was used as the unit of measurement.

Statistical analysis. The data obtained by immunohistochemistry were subjected to statistical analysis using one-way analysis of variance followed by Tukey's post-hoc test. Data are expressed as the mean \pm standard error of the mean, with each experiment repeated three times. $\mathrm{P}<0.05$ was considered to indicate a statistically significant difference. All statistical tests were performed using GraphPad Prism software (version 4.00; GraphPad Software, Inc., La Jolla, CA, USA).

\section{Results}

Phenotypic characteristics of implanted cells. As indicated in Fig. 1, the majority of the C6 glioma specimens $(96.0 \pm 6.8 \%)$ stained positive for antibodies against nestin and mutant protein p53 (88.4 $\pm 3.8 \%)$. A marked proportion of tumor cells expressed CXCR4 receptor $(62.4 \pm 8.3 \%)$. However, the proportion of cells with antigen properties of glial cells (nestin-negative; S100- and GFAP-positive) or neural differentiation was not substantial $(1.0 \pm 0.1 \%$; Fig. $1 \mathrm{~A}-\mathrm{C})$.

Tumor morphology. An MRI scan performed 10 days after the implantation of tumor cells showed massive tumors (mean \pm SEM, $5.6 \pm 1.4 \mathrm{~mm}^{3}$ ) in the brains of all the animals. Morphological analysis revealed that the tumor was comprised of variously shaped cells with different numbers of nuclei of different sizes. By day 20, the neoplastic tissue contained a large number blood microvessels, indicating a high metabolism. At 
A

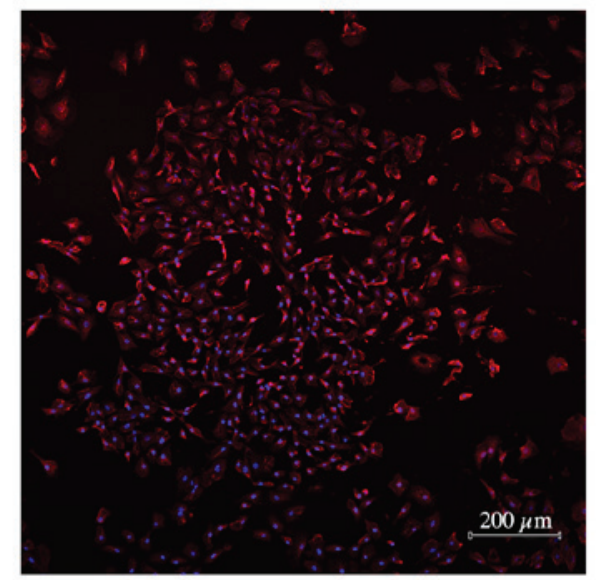

B

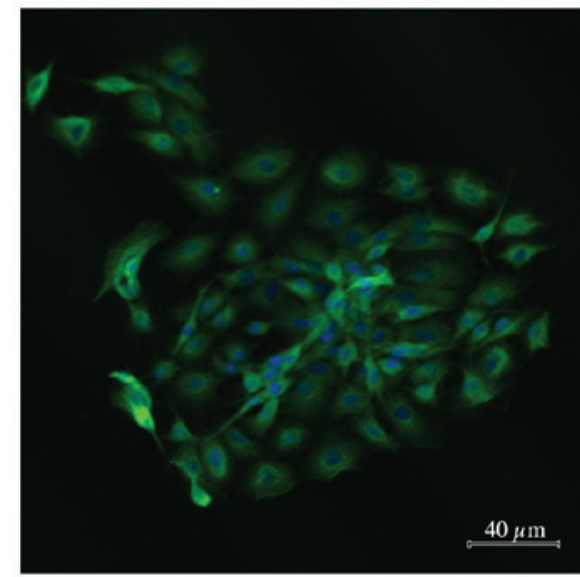

C

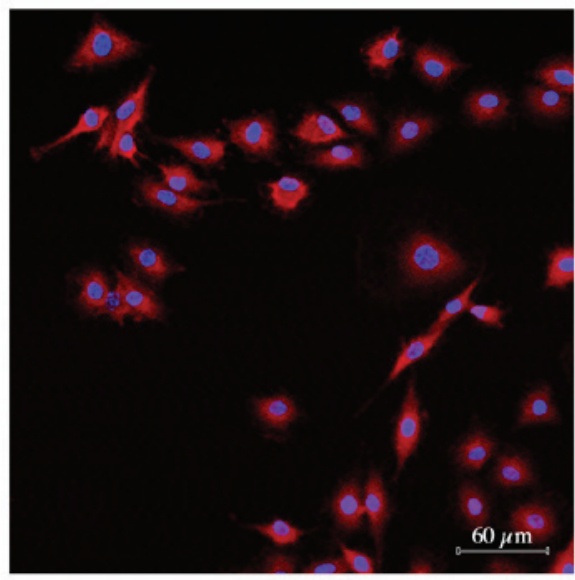

Figure 1. Immunocytochemical characteristic of glioma cells used in the experiment. Antibody staining against (A) nestin, (B) p53 and (C) C-X-C chemokine receptor type 4. Cytoplasms are stained with (A and C) Alexa 633 (pink) or (B) Alexa 488 (green). Nuclei are counterstained by DAPI (blue).

the periphery of the tumor nidus, the glioma cells invaded the brain parenchyma with dystrophic changes and the creation of chords surrounded by a large number of cells (Fig. 2A). A little farther from the primary nodule, the glioma cells were clustered into conglomerates creating secondary satellite tumors. Rapid growth of a feeding blood vessel in the center of the secondary nidus intensified neoplastic development and invasive processes (Fig. 2B).

On days 20-30, microscopic observation identified the start of tumor cell death. Necrosis observed in the center of the tumor nodule close to the feeding blood vessels indicated that the blood system was unable to satisfy the needs of the fast-multiplying cells. By day 30, the tumor cells created thick nodule-like clusters around the feeding blood vessel showing a typical 'roseola' pattern (Fig. 2C) with necrotic areas occupying the main space of the morphological images (Fig. 2D). The mean life expectancy of the experimental animals was $34.0 \pm 8.1$ days.

Tumor immunocytochemistry. PCNA antibody staining was used to indicate the extent of proliferation (Fig. 3A-D). PCNA staining revealed a sharp increase in the proliferation speed of the tumor nodule on day 20 compared with day 10 that may be associated with the intensification of tumor cell division due to the development of the neoplastic blood supply network (Fig. 3A and D). Outside the tumor nidus, the maximum proportion of PCNA-positive cells was concentrated in the adjacent tissues, satellite nodules and blood vessels (data not shown). By day 30, the number of proliferating cells in the tumor tissue significantly decreased (Fig. 3B and D). Furthermore, by day 20, PCNA-positive cells had been located along the tumor borders in areas of the brain with the maximum infiltration of glioma cells due to their invasive growth (Fig. 3C). Stand-alone proliferating cells were observed in the brain tissue a significant distance from the tumor nidus (data not shown).

The maximum concentration of microglial cells was observed in the tumor tissue itself and adjacent glioma invasion areas on days 10-20, as opposed to in tissues from the opposite hemisphere of the brain (Fig. 4A-F). Significant clusters of small IBA-1-positive cells with an elongated shape and short extensions were concentrated in the brain parenchyma a small distance from the tumor nodule and in the endothelium of hypertrophied blood vessels (Fig. 4B). The changes in microglial cell concentration in the neoplastic nodule between days 10 and 20 may be caused by the transformation of monocytes migrating from the newly formed capillaries into resident macrophages, as well as by the local migration of microglia from the adjacent brain tissues. The number of IBA-1-positive cells in the brain parenchyma of the hemisphere containing the tumor was significantly larger than the number in the intact (opposite) hemisphere 20 days after implantation (Fig. 4C and F).

After day 20, the number of microglial cells in the tumor nodus began decreasing. Cells of round and amoeboid shape were visible in parabasal concentrations of glioma cells, creating thick lines around necrotic areas (Fig. 4D). In addition, by day 30 , a marked number of microglial cells were localized along the borders of the tumor nidus, particularly in areas of glioma invasion into the brain parenchyma. By day 30 , the microglial cells were almost absent from the tumor nodule tissue but were instead forming a rim of tumor cells (Fig. 4E and F).

The aforementioned results are due to the complex and specific interaction of microglia with tumor cells and CSCs, and are associated with the secretion of certain cytokines. On day 30, the areas of perifocal invasion with the maximum concentration of microglial cells were stained with antibodies against IL-1 $\beta$, nestin, CXCR4 and TGF-2 $\beta$. The tumor and neartumor areas were heavily infiltrated with nestin; this indicated the presence of poorly differentiated cells, either CSCs or neural SCs, which, as demonstrated by the high CXCR4 expression in this location, may have migrated here. Increased expression of IL-1 $\beta$ was present within the tumor, and TGF- $\beta$ expression was demonstrated in the near-tumor areas (Fig. 5A-D). 

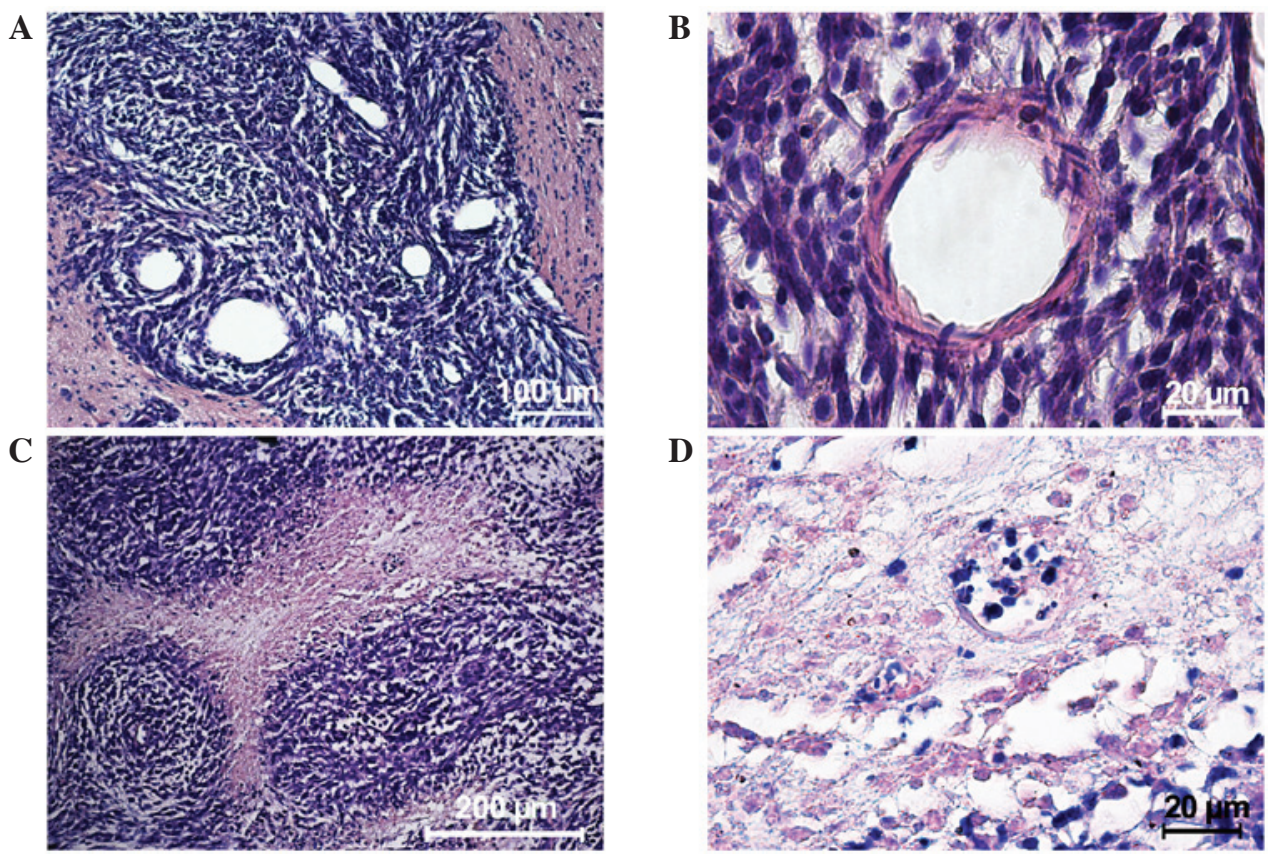

Figure 2. Hematoxylin and eosin staining of neoplastic tissue in the rat brain. Twenty days after implantation, (A) newly formed blood vessels were observed in the tumor tissue and (B) a blood vessel developed among tumor cells of the satellite nodule, distant from the primary nodule. Thirty days after implantation, (C) an angiocentric cluster of tumor cells developed and (D) necrotic areas were observed in the tumor tissue.
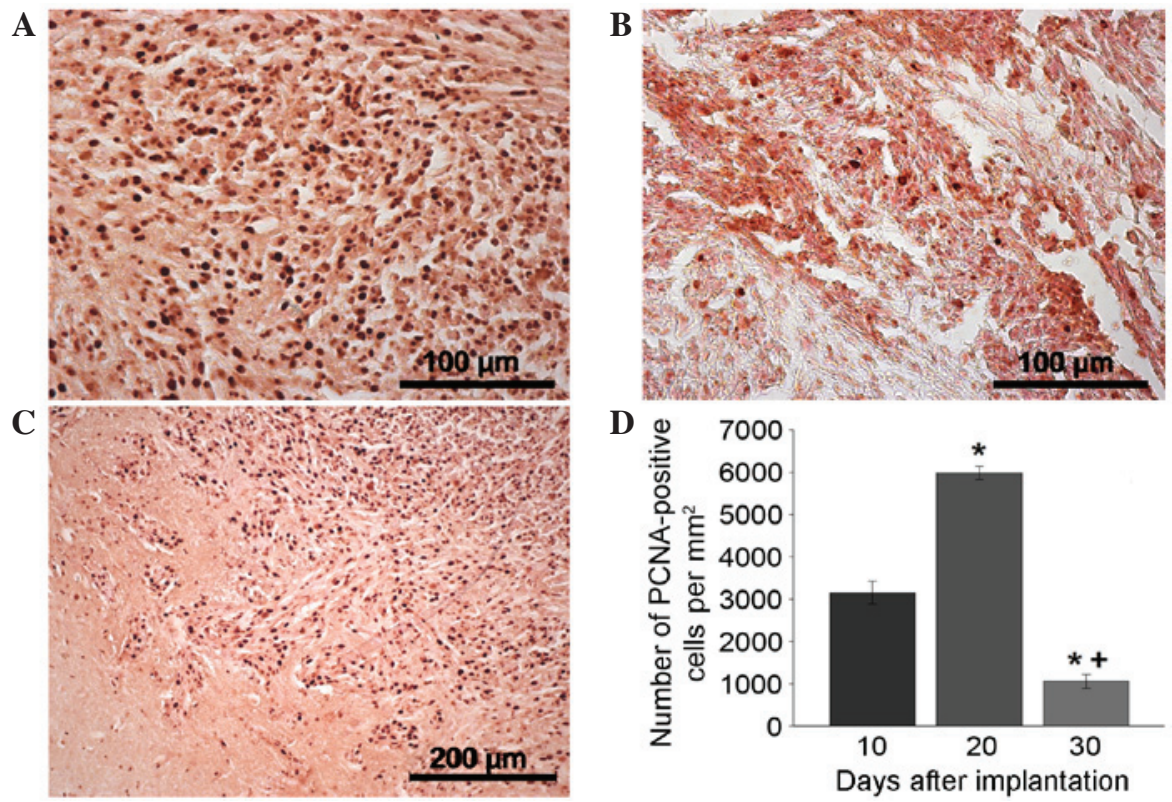

Figure 3. Tumors in the rat brain. Immunocytochemical antibody staining for PCNA in the neoplastic nodule (A) 20 and (B) 30 days after implantation. (C) Area of tumor invasion into the brain parenchyma on day 20 after implantation. (D) PCNA-positive cell performance dynamics in the neoplastic nodule over time. ${ }^{*} \mathrm{P}<0.05$ vs. 10 days; ${ }^{+} \mathrm{P}<0.05$ vs. 20 days. PCNA, proliferating cell nuclear antigen.

\section{Discussion}

CSCs are the driving force of glioblastoma growth and one of the primary causes of therapeutic resistance in cancer. The basic properties of CSCs allow quick proliferation and the establishment of angiogenesis, allowing fast accumulation of the necessary resources. This notion is confirmed by the present PCNA staining results. In addition, CSCs occupy the leading position in the tissue hierarchy, over other somatic cells and SCs, and extend neoplastic processes into adjacent tissues (14).
The glioma cells used in the current study are immunopositive for nestin, one of the key markers of neural SCs. Nestin is a selective marker of highly malignant tumors, including breast cancer of the basal epithelium, melanoma and glioblastoma. In addition, CXCR4 protein is one of the major markers for different types of SCs, including CSCs (15). C6 glioma cells have a mutation in the most important tumor suppressor in humans, the $\mathrm{p} 53$ gene. This mutation means that $\mathrm{C} 6$ glioma cells are categorized as a mutant cell line with immunophenotypic properties of SCs. In the present study, single-step implantation 
A

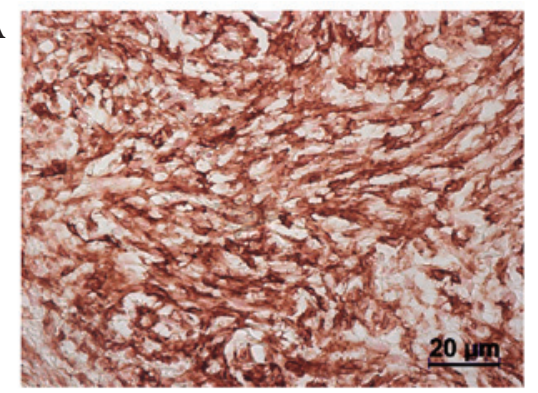

C

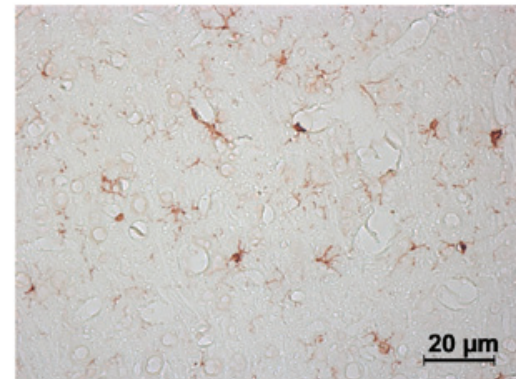

$\mathbf{E}$

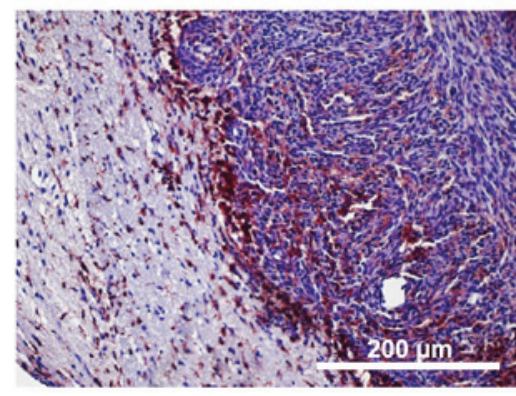

B

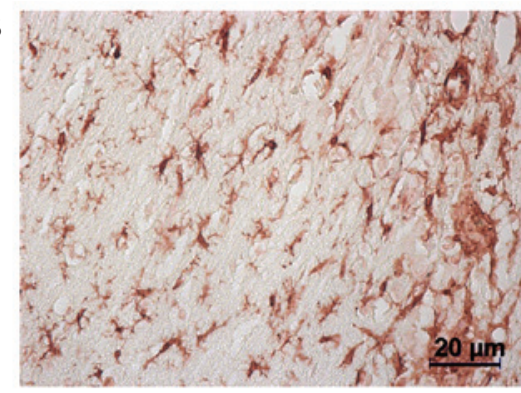

D

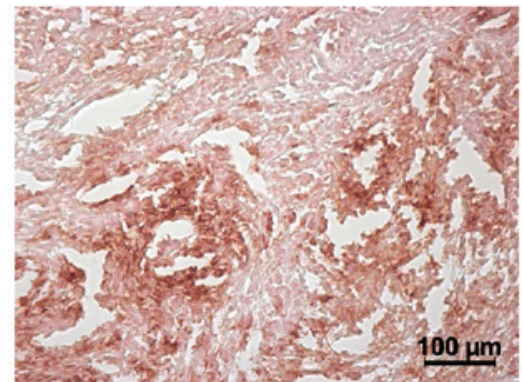

$\mathbf{F}$

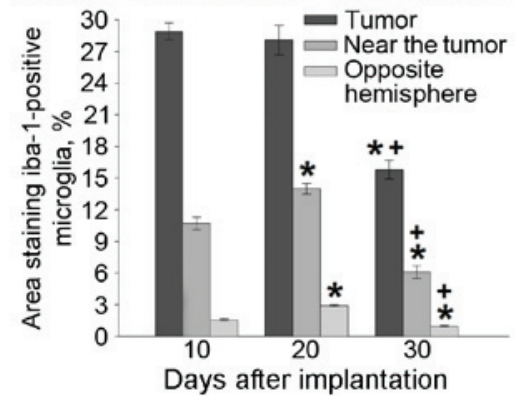

Figure 4. Tumor in the rat brain. Immunocytochemical antibody staining for IBA-1 (microglia/macrophage-specific protein) in (A) the neoplastic nodule, (B) the brain area adjacent to the nodule and (C) the brain area of the hemisphere opposite to the nodule 20 days after implantation, and in (D) the neoplastic nodule 30 days after implantation. (E) Staining with hematoxylin and eosin and IBA-1 antibody revealed IBA-positive cells on the border of the neoplastic nodule on day 30 after implantation. (F) IBA-1-positive cell performance dynamics in neoplastic nodule over time ${ }^{*} \mathrm{P}<0.05$ vs. 10 days; ${ }^{+} \mathrm{P}<0.05$ vs. 20 days. IBA-1, ionized calcium-binding adapter molecule-1.

of $0.2 \times 10^{6} \mathrm{C} 6$ glioma cells into a rat brain stimulated the rapid development of invasive tumors with high proliferation rates.

High proliferation speed is a major contributing factor to the neoplastic process of glioma cells. By day 20 of the current study, the tumor cell proliferation rate was faster than the angiogenesis rate (data not shown), resulting in the generation of a hypoxic microenvironment. Massive hypoxic death of tumor cells stimulates the production of hypoxia-inducible factor molecules, whose major targets are angiogenesis-associated genes. Thus, by stimulating the production of angiogenic genes, such as vascular endothelial growth factor, hypoxia completes the circle and contributes to the selection of the most resistant clones of tumor cells (16).

Hypoxia has a multifunctional effect. The low partial pressure of oxygen prevents the creation of active radicals, significantly reducing the effects of radiation and creating optimum conditions for CSC survival. Hypoxia is an important factor involved in the expression of genes responsible for the biosynthesis of cytokines, which initiate the directed migration of somatic cells and SCs into a tumor (17).

Microglia consist of $30 \%$ of cells recruited to the tumor environment $(18,19)$. The main factor in this process is stromal cell-derived factor $1 \alpha$ (also known as C-X-C motif chemokine 12). The ligand is released as a response to hypoxic damage of tissues and actively attracts SCs of all types, including monocytes, into the neoplastic area where they can be transformed into microphages and microgliocytes (20). Another important inducer of SC and monocyte migration to the tumor nidus is monocyte chemoattractant protein-1; this ligand was initially identified in malignant glioma (21).

Microglial cells form complex secretory units by interacting with CSCs in the neoplastic nidus. For example, highly malignant glioma cells produce colony stimulating factor-1, attracting microglia and microphages that secrete epidermal growth factor (EGF). EGF accelerates the rate of proliferation, and induces the production of matrix metalloproteinase (MMP)2, 9 and 14 from tumor cells, causing the destruction of tissue barriers and the extension of neoplastic processes into adjacent tissues (22).

The tumor modeled in the current study was characterized by excessive production of cytokines, as indicated by the significant number of microglial cells concentrated in the tumor nidus itself and the adjacent tissues. However, as the tumor cells began to die due to hypoxia, the number of microglia and macrophages in the tumor tissue decreased, as evidenced by the continuous IBA-1-positive cell number reduction. Subsequently, microglial cells were concentrated along the border of the tumor nidus in the area of glioma cell 

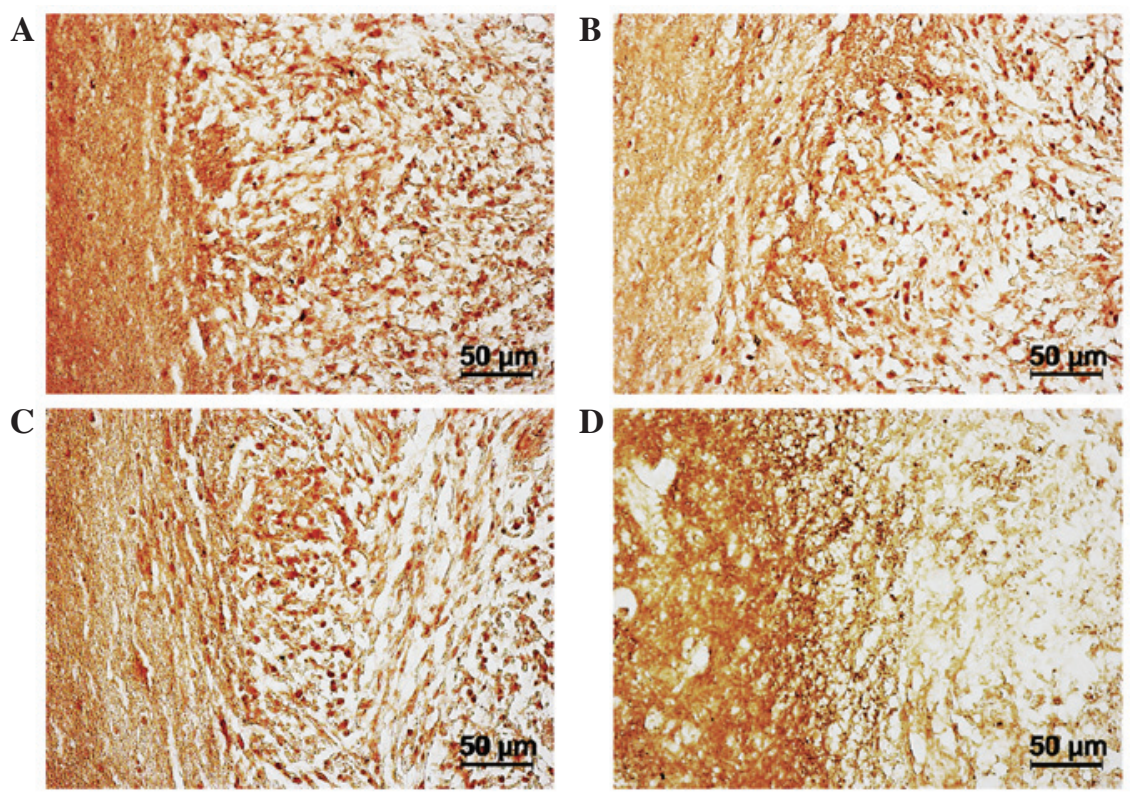

Figure 5. Border of neoplastic nodule in the rat brain 30 days after implantation. Immunocytochemical antibody staining against (A) nestin, (B) C-X-C chemokine receptor type $4,(C)$ interleukin-1 $\beta$ and (D) transforming growth factor- $2 \beta$.

invasion into the brain parenchyma. It is possible that, in this case, hypoxia was an important inductor of recruited cell traffic, but not the main controller.

The concentration of microglia at the tumor periphery may be explained by the influence of the specific local microenvironment created by the CSCs. The presence of CSCs in the neoplastic invasion area was demonstrated by local positive staining for nestin and CXCR4 antibody. CXCR4 and nestin expression were noted in a significant proportion of glioma cells, indicating that these cells have CSC functions. Furthermore, CXCR4 is a membrane component of microglial cells and monocytes that are attracted by the tumor. Thus, there is a high probability that the microenvironment created by CSCs selectively activates microglia with an M2 (anti-inflammatory) phenotype, facilitating tumor growth and invasive processes (23).

On days 20-30 of the current study, areas of concentrated microglial cells were observed along the tumor border and in adjacent parts of the nervous tissue by staining with antibodies against IL-1 $\beta$. This cytokine is able to induce NO synthetase, leading to excessive NO formation that can damage neurons and increase hypoxia (24).

Notably, TGF- $\beta$-immunopositive cells were concentrated in the area of tumor invasion in the present study. TGF- $\beta$ is one of the main ligands responsible for the invasive properties of glioblastoma; it triggers epithelial-mesenchymal transition in glioma cells, causing cells to a obtain locomotor phenotype and induce MMP synthesis. A previous study revealed that TGF- $\beta$ is able to cause metastasis and contributes to tumor development via an autocrine mechanism. TGF- $\beta$ produced by microglia intensifies glioma cell proliferation and invasive processes (25). In addition, the TGF- $\beta$ signaling pathway, as well as Notch and Sonic Hedgehog cascades, is a key controllers of CSC proliferation functions (26).

Thus, after being implanted into the brain, CSCs are able to trigger proliferation that creates a hypoxic microenvironment, inducing directed migration of different somatic cells and SCs that are necessary for angiogenesis and proteolysis. A substantial proportion of cells recruited by the tumor are microglial cells that have a strategic role in invasive growth. TGF- $\beta$ and other ligands produced by the microglia trigger epithelial-mesenchymal transition in glioma cells, intensifying CSC proliferative functions and creating an immunosuppressive medium that facilitates the invasion process.

SCs migrating into neoplastic tissue are able to have a direct anti-tumor effect. The mechanism of this effect is SC stimulation of the Akt and Wnt signaling pathways $(27,28)$, tumor cell cycle arrest in the G1 phase or direct induction of apoptosis (29). This property also means that SCs could be utilized to deliver medication directly to the tumor nidus or to release specific ligands that interact with major pathways of signal transduction in CSCs, suppressing the production of cytokines stimulating invasive growth by microglial cells.

The production of cytokines by microglial cells to intensify invasive processes is suppressed by activating the signal transducer and activator of transcription 3 signaling pathway (11) and p38 mitogen-activated protein kinase (30). Currently, research is aimed at identifying novel substances that can activate these signaling pathways. Using minocycline and doxycycline suppresses tumor microglia activity by blocking MMP synthesis (31). However, there is a more promising method of regulating microglia and CSC function: By using cell adhesion receptors to stimulate integrins.

Integrins have a key role in CSC remodeling of the local microenvironment that occurs during invasive growth. Suppressing integrin expression reduces the size of a tumor in vivo and reduces microglial cell density at the border of a glioma (32).

In 2014, our previous study reported that the focal adhesion pathway in CSCs of the U87 human glioblastoma cell line is safe and that it is possible to stimulate this pathway with SC-based medication with a modified transcriptomic profile (6). Microglia have a strategically important role in 
promoting invasive growth, as they are concentrated in the areas with the highest activity of CSCs that are the primary target of biomedical cell-based treatment. We propose that changes in microglia and subsequent tumor growth suppression may be important criteria in evaluating the effectiveness of cell-based treatment for glioblastoma in vivo.

In summary, CSCs are among the leading reasons behind therapeutic resistance in glioblastoma. Currently there are no effective ways to eliminate CSCs within the patient's body. Development of biomedical approaches based on autologous $\mathrm{SCs}$ is a possible way to resolve this problem. Regional human SCs hold high antitumor potential. These migrate towards cancer and accumulate in the neoplastic tissue. We hypothesized that the most likely direction of SC differentiation within cancer foci is into elements of cancer microglia.

The present work describes the in vivo dynamics of microgliacyte distribution during invasive glial tumor growth. The findings demonstrated that during glioblastoma development, microglia localization corresponds to nestin-positive, CSC-rich areas of active tumor cell proliferation and invasion. Active production of TGF- $\beta$ and IL- 1 within this area indicates of the presence of complex regulatory relationships between interacting cells which, in theory, could be controlled by transplanting modified SCs. Changes in microglia localization and concomitant inhibition of tumor growth can be used for assessment of antitumor therapy effectiveness.

\section{Acknowledgements}

The present study was funded by the Ministry of Education and Science of the Russian Federation (project no., 14.575.21.0038; project ID, RFMEF157514X0038).

\section{References}

1. Omuro A and DeAngelis LM: Glioblastoma and other malignant gliomas: A clinical review. JAMA 310: 1842-1850, 2013.

2. Louis DN, Perry A, Burger P, Ellison DW, Reifenberger G, von Deimling A, Aldape K, Brat D, Collins VP, Eberhart C, et al: International society of neuropathology-haarlem consensus guidelines for nervous system tumor classification and grading. Brain Pathol 24: 429-435, 2014.

3. Stupp R, Mason WP, van den Bent MJ, Weller M, Fisher B, Taphoorn MJ, Belanger $\mathrm{K}$, Brandes AA, Marosi C, Bogdahn U, et al: Radiotherapy plus concomitant and adjuvant temozolomide for glioblastoma. N Engl J Med 352: 987-996, 2005.

4. Cruceru ML, Neagu M, Demoulin JB and Constantinescu SN: Therapy targets in glioblastoma and cancer stem cells: Lessons from hematopoietic neoplasms. J Cell Mol Med 17: 1218-1235, 2013.

5. Bryukhovetskiy I, Bryukhovetsky A, Khotimchenko Y, Mischenko P, Tolok E and Khotimchenko R: Combination of the multipotent mesenchymal stromal cell transplantation with administration of temozolomide increases survival of rats with experimental glioblastoma. Mol Med Rep 12: 2828-2834, 2015.

6. Bryukhovetskiy A, Shevchenko V, Kovalev S, Chekhonin V, Baklaushev V, Bryukhovetskiy I and Zhukova M: To the novel paradigm of proteome-based cell therapy of tumors: Through comparative proteome mapping of tumor stem cells and tissue-specific stem cells of humans. Cell Transplant 23 (Suppl 1): S151-S170, 2014.

7. Saraceni F, Shem-Tov N, Olivieri A and Nagler A: Mobilized peripheral blood grafts include more than hematopoietic stem cells: The immunological perspective. Bone Marrow Transplant 50: 886-891, 2015.

8. Syme R, Bajwa R, Robertson L, Stewart D and Glück S: Comparison of CD34 and monocyte-derived dendritic cells from mobilized peripheral blood from cancer patients. Stem Cells 23: 74-81, 2005.
9. Moore XL, Lu J, Sun L, Zhu CJ, Tan P and Wong MC: Endothelial progenitor cells 'homing' specificity to brain tumors. Gene Ther 11: 811-818, 2004.

10. Ye XZ, Xu SL, Xin YH, Yu SC, Ping YF, Chen L, Xiao HL, Wang B, Yi L, Wang QL, et al: Tumor-associated microglia/macrophages enhance the invasion of glioma stem-like cells via TGF- $\beta 1$ signaling pathway. J Immunol 189: 444-453, 2012.

11. da Fonseca AC and Badie B: Microglia and macrophages in malignant gliomas: Recent discoveries and implications for promising therapies. Clin Dev Immunol 2013: 264124, 2013.

12. Barth RF and Kaur B: Rat brain tumor models in experimental neuro-oncology: The C6, 9L, T9, RG2, F98, BT4C, RT-2 and CNS-1 gliomas. J Neurooncol 94: 299-312, 2009.

13. Paxinos G and Watson C: The Rat Brain in Stereotaxic Coordinates. 6th edition. Elsevier Academic Press, San Diego, 2007.

14. Lathia JD, Mack SC, Mulkearns-Hubert EE, Valentim CL and Rich JN: Cancer stem cells in glioblastoma. Genes Dev 29: 1203-1217, 2015.

15. Trautmann F, Cojoc M, Kurth I, Melin N, Bouchez LC, Dubrovska A and Peitzsch C: CXCR4 as biomarker for radioresistant cancer stem cells. Int J Radiat Biol 90: 687-699, 2014.

16. Zhao D, Najbauer J, Garcia E, Metz MZ, Gutova M, Glackin CA, Kim SU and Aboody KS: Neural stem cell tropism to glioma: Critical role of tumor hypoxia. Mol Cancer Res 6: 1819-1829, 2008.

17. Wierenga AT, Vellenga E and Schuringa JJ: Convergence of hypoxia and TGF $\beta$ pathways on cell cycle regulation in human hematopoietic stem/progenitor cells. PLoS One 9: e93494, 2014.

18. Held-Feindt J, Hattermann K, Müerköster SS, Wedderkopp H, Knerlich-Lukoschus F, Ungefroren H, Mehdorn HM and Mentlein R: CX3CR1 promotes recruitment of human glioma-infiltrating microglia/macrophages (GIMs). Exp Cell Res 316: 1553-1566, 2010.

19. Coniglio SJ, Eugenin E, Dobrenis K, Stanley ER, West BL, Symons MH and Segall JE: Microglial stimulation of glioblastoma invasion involves epidermal growth factor receptor (EGFR) and colony stimulating factor 1 receptor (CSF-1R) signaling. Mol Med 18: 519-527, 2012.

20. Greenbaum A, Hsu YM, Day RB, Schuettpelz LG, Christopher MJ, Borgerding JN, Nagasawa T and Link DC: CXCL12 in early mesenchymal progenitors is required for haematopoietic stem-cell maintenance. Nature 495: 227-230, 2013.

21. Desbaillets I, Tada M, de Tribolet N, Diserens AC, Hamou MF and Van Meir EG: Human astrocytomas and glioblastomas express monocyte chemoattractant protein-1 (MCP-1) in vivo and in vitro. Int J Cancer 58: 240-247, 1994.

22. Markovic DS, Vinnakota K, Chirasani S, Synowitz M, Raguet H, Stock K, Sliwa M, Lehmann S, Kälin R, van Rooijen N, et al: Gliomas induce and exploit microglial MT1-MMP expression for tumor expansion. Proc Natl Acad Sci USA 106: 12530-12535, 2009.

23. Wu A, Wei J, Kong LY, Wang Y, Priebe W, Qiao W, Sawaya R and Heimberger AB: Glioma cancer stem cells induce immunosuppressive macrophages/microglia. Neuro Oncol 12: 1113-1125, 2010.

24. Hu Y, Xue J, Yang Y, Zhou X, Qin C, Zheng M, Zhu H, Liu Y, Liu W, Lou G, et al: Lipocalin 2 upregulation protects hepatocytes from IL1- $\beta$-induced stress. Cell Physiol Biochem 36: 753-762.

25. Ikushima $\mathrm{H}$, Todo $\mathrm{T}$, Ino $\mathrm{Y}$, Takahashi $\mathrm{M}$, Miyazawa $\mathrm{K}$ and Miyazono K: Autocrine TGF-beta signaling maintains tumorigenicity of glioma-initiating cells through Sry-related HMG-box factors. Cell Stem Cell 5: 504-514, 2009.

26. Caja L, Bellomo $\mathrm{C}$ and Moustakas A: Transforming growth factor $\beta$ and bone morphogenetic protein actions in brain tumors. FEBS Lett 589: 1588-1597, 2015.

27. Qiao L, Xu Z,Zhao T, Zhao Z, Shi M, Zhao RC, Ye L and Zhang X: Suppression of tumorigenesis by human mesenchymal stem cells in a hepatoma model. Cell Res 18: 500-507, 2008.

28. Moreno E: Cancer: Darwinian tumour suppression. Nature 509: 435-436, 2014.

29. Cousin B, Ravet E, Poglio S, De Toni F, Bertuzzi M, Lulka H, Touil I, André M, Grolleau JL, Péron JM, et al: Adult stromal cells derived from human adipose tissue provoke pancreatic cancer cell death both in vitro and in vivo. PLoS One 4: e6278, 2009.

30. Zhang Y, Ren X, Shi M, Jiang Z, Wang H, Su Q, Liu Q, Li G and Jiang G: Downregulation of STAT3 and activation of MAPK are involved in the induction of apoptosis by HNK in glioblastoma cell line U87. Oncol Rep 32: 2038-2046, 2014.

31. Sun JS, Yang YJ, Zhang YZ, Huang W, Li ZS and Zhang Y: Minocycline attenuates pain by inhibiting spinal microglia activation in diabetic rats. Mol Med Rep 12: 2677-2682, 2015.

32. Färber K, Synowitz M, Zahn G, Vossmeyer D, Stragies R, van Rooijen N and Kettenmann H: An alpha5betal integrin inhibitor attenuates glioma growth. Mol Cell Neurosci 39: 579-585, 2008. 